\title{
Dislipidemias em Crianças e Adolescentes. Bases para a Terapêutica
}

\author{
Neusa Forti, Jaqueline Issa, Jayme Diament, Sergio Diogo Giannini
}

São Paulo, SP

Estudos anatomopatológicos, epidemiológicos, experimentais, clínicos e terapêuticos têm demonstrado exaustivamente a existência do vínculo entre a presença de alterações do metabolismo lipídico e doença aterosclerótica, particularmente a das artérias coronárias (DAC).

As estrias gordurosas, consideradas precursoras das placas ateroscleróticas, começam a aparecer na aorta aos três anos de idade e, aos 15 anos, comprometem $15 \%$ dessa artéria. Nas coronárias, elas surgem a partir dos 15 anos de idade ${ }^{1}$.

Na investigação realizada em Bogalusa ${ }^{2}$, verificou-se que, em crianças e adolescentes (idade variando entre sete e 24 anos, média 18 anos), falecidas durante o estudo, o comprometimento da aorta por estrias gordurosas mostrouse significativamente maior quando os níveis plasmáticos de colesterol total (CT) e LDL-C (determinados durante o estudo) e/ou o peso corpóreo estavam aumentados. Nas artérias coronárias, as estrias gordurosas também foram mais freqüentes na presença de níveis aumentados de CTe LDL-C (embora não significante); nos brancos e do sexo masculino, apresentavam-se significativamente associados aos valores de triglicérides (TG), VLDL-C, pressão arterial sistólica e diastólica e índice de massa corpórea. Em estudo multicêntrico ${ }^{3}$, em jovens de 15 a 34 anos (1079 do sexo masculino e 364 do feminino) falecidos em consequiência de acidentes, homicídios e suicídios, foi verificada correlação significante entre valores aumentados de LDL-C e VLDL-C e diminuídos de HDL-C (determinados após a morte) e a extensão da severidade de lesões tanto na aorta como na coronária direita.

Manifestações clínicas (infarto do miocárdio, angina, morte súbita) conseqüentes a lesões ateroscleróticas são comuns antes dos 20 anos nos estados hipercolesterolêmicos familiares, particularmente na forma homozigótica ${ }^{4}$. Evidências de disfunção endotelial em hipercolesterolêmicos familiares, de oito a 16 anos, foram verificadas por Celermajere $\mathrm{col}^{5}$.

Por outro lado, dados de estudos prospectivos são indicativos de que alterações do metabolismo lipídico, presentes na infância e adolescência, persistem na idade adul-

Instituto do Coração do Hospital das Clínicas - FMUSP

Correspondência: Neusa Forti - Incor - Av. Dr. Enéas C. Aguiar, 44 - andar AB (anexo) - 05403-000 - São Paulo, SP

Recebido para publicação em 29/6/98

Aceito em22/7/98 ta, constituindo-se em importante fator de risco para o desenvolvimento das lesões ateroscleróticas. Resultados das investigações de Bogalusa ${ }^{6}$ e Muscatine ${ }^{7}$ demonstram que aproximadamente 50\% das crianças com CT acima do percentil 75 (da curva de distribuição do Lipid Research Clinic) apresentarão valores de LDL-C elevados 10 a 15 anos mais tarde. No estudo de Bogalusa ${ }^{6}$ também se verificou que $42 \%$ dos meninos brancos ( 9 a 14 anos), que apresentavam valores de HDL-C no quintil mais baixo, continuavam a apresentá-los nesses valores 12 anos mais tarde. Crianças e adolescentes com valores de TG $>100$ e $130 \mathrm{mg} / \mathrm{dL}$, respectivamente, e história familiar de DAC prematura, podem ser portadores de gens para dislipidemias genéticas, como, por exemplo, para a hiperlipidemia combinada familiar ${ }^{8}$.

Levando em consideração a indiscutível participação das dislipidemias na aterogênese e diante do já exposto, é lícito inferir que há necessidade de identificar e tratar as hiperlipidemias o mais precocemente possível para prevenir e/ou retardar o aparecimento de lesões ateroscleróticas. Surgem, então, duas indagações: 1) o perfil lipídico deve ser determinado em todas as crianças e adolescentes?; 2) como orientar o tratamento?

Fundamentado em consensos e recomendações de outros países 9,10, o Consenso Brasileiro sobre Dislipidemias ${ }^{11}$ considera que a determinação do perfil lipídico mínimo(CT, TG, LDL-CeHDL-C) deve ser realizada em crianças e adolescentes quando: 1) avós, pais, irmãos, tios e primos de $1^{\circ}$ grau tenham tido prematuramente manifestação de DAC (antes dos 55 anos para o sexo masculino ou dos 65 anos para o feminino); 2) quando algum desses parentes próximos apresentarem $\mathrm{CT} \geq 300$ ou $\mathrm{TG} \geq 400 \mathrm{mg} / \mathrm{dL}$; 3 ) houver presença de xantomatose ou de outros fatores de risco para DAC (obesidade, hipertensão arterial, diabetes mellitus, sedentarismo, tabagismo); 4) houver manifestação de pancreatite ou de DAC. Recomenda-se que o perfil lipídico seja determinado duas vezes, com intervalo de oito a 15 dias, mantendo-se as mesmas condições de coleta ${ }^{11}$. Os valores de referência estão expostos na tabela I.

Com base nesses valores, análise do aspecto do soro e dados de observação clínica, diferentes tipos de dislipidemias podem ser identificados na infância e na adolescência (quadro I). Na prática, encontram-se mais freqüentemente a hipercolesterolemia e a hipertrigliceridemia comuns ou ambientais, isto é, aquelas que são causadas por múltiplos fatores genéticos e/ou ambientais ${ }^{11}$. 


\begin{tabular}{|c|c|c|c|c|}
\hline \multicolumn{5}{|c|}{$\begin{array}{l}\text { Tabela I - Valores de referência de variáveis do perfil lipídico em } \\
\text { crianças e adolescentes (entre } 2 \text { e } 19 \text { anos de idade) }{ }^{11}\end{array}$} \\
\hline \multirow[t]{2}{*}{ Lípides } & \multirow[t]{2}{*}{ Idade (anos) } & \multicolumn{3}{|c|}{ Valores $(\mathrm{mg} / \mathrm{dL})$} \\
\hline & & Desejáveis & Limítrofes & Aumentados \\
\hline $\mathrm{CT}$ & & $<170$ & $170-199$ & $\geq 200$ \\
\hline LDL-C & & $<110$ & $110-129$ & $\geq 130$ \\
\hline \multirow[t]{2}{*}{ HDL-C* ${ }^{*}$} & $<10$ & $\geq 40$ & --- & --- \\
\hline & $10-19$ & $\geq 35$ & --- & --- \\
\hline \multirow{2}{*}{$\mathrm{TG}^{*}$} & $<10$ & $\leq 100$ & --- & $>100$ \\
\hline & $10-19$ & $\leq 130$ & --- & $>130$ \\
\hline
\end{tabular}

\begin{tabular}{|l|}
\hline \multicolumn{1}{|c|}{$\begin{array}{c}\text { Quadro I - Principais tipos de dislipidemias identificadas na } \\
\text { infância e adolescência }{ }^{\text {9-11 }}\end{array}$} \\
\hline Primárias \\
• Hipercolesterolemia ambiental (II a) \\
- Hipercolesterolemia familiar homo ou heterozigótica (II a) \\
- Hipertrigliceridemia ambiental (IV) \\
- Quilomicronemia (I) \\
- Hiperlipidemia combinada familiar (II a, II b, IV) \\
- Disbetalipoproteinemia (III) \\
- Hipertrigliceridemia e quilomicronemia (V) \\
Secundárias \\
- diabetes mellitus \\
- hipotireoidismo \\
- síndrome nefrótica \\
\hline Obs.: os números em algarismos romanos colocados entre parênteses \\
correspondem à classificação de Fredrickson, referendada pela OMS. \\
\hline
\end{tabular}

No Brasil, há poucos estudos sobre a prevalência de desvios lipídicos na infância e adolescência. Em 1502 escolares, de seis a 12 anos, residentes em Bento Gonçalves (Rio Grande do Sul), Gerber ${ }^{12}$ verificou colesterolemia $>180 \mathrm{mg} / \mathrm{dL}$ em 27,9\%, níveis de LDL-C >130mg/dL em 10,3\%, trigliceridemia $>140 \mathrm{mg} / \mathrm{dL}$ em $9,5 \%$. No programa de prevenção primária de DAC em desenvolvimento no Instituto do Coração do HCFMUSP, foi analisado, entre os diferentes fatores de risco, perfil lipídico de 104 crianças (dois a 12 anos, mediana 9 anos, 48 meninos e 58 meninas) e de 180 adolescentes (12 a 19 anos, mediana 16 anos, 85 do sexo feminino e 91 do masculino), considerando-se os valores ideais preconizados pelo Consenso Brasileiro sobre Dislipidemias ${ }^{11}$. Observaram-se respectivamente, nas crianças e adolescentes, valores indesejáveisdeCTem57,7\% e 42,7\%, deLDL-Cem55,4\% e 38,3\%, de TGem 51,0\% e 31,9\%, de HDL-Cem 13,5\% e 14,2\%.Esses desvios estiveram relacionados à presença de obesidade e sobrepeso. Somente foram identificados quatro casos de hipercolesterolemia familiar heterozigótica e dois casos de hipertrigliceridemiafamiliar ${ }^{13}$.

\section{Terapêutica para dislipidemias primárias}

Recomenda-se seja iniciada após os dois anos de idade e tem por objetivo alcançar os níveis ideais preconizados pelo Consenso Brasileiro sobre Dislipidemias ${ }^{11}$, reduzir xantomatose, diminuir os riscos de pancreatite e prevenir o aparecimento de $\mathrm{DAC}$ e, quando presente, o que é raro, promover a estabilização e regressão das lesões existentes ${ }^{9,11,14}$.

Fundamenta-se na adoção de padrões alimentares adequados, de acordo com o desvio lipídico apresentado, mantendo-se a ingestão de vitaminas e a quantidade de calorias necessárias para o desenvolvimento e crescimento da criança ou adolescente. Para tanto, devem ser avaliados os hábitos individuais e familiares. Para melhor conduzir a orientação dietética, torna-se valiosa a colaboração do pediatra e do nutricionista.

Paralelamente, estimula-se a prática de exercícios físico aeróbicos e controlam-se outros eventuais fatores de risco. Medicamentos e medidas alternativas são introduzidos em situações especiais ${ }^{9,11,14}$.

Orientação dietética - Para a hipercolesterolemia, recomenda-se a diminuição da ingestão de alimentos ricos em colesterol e em ácidos graxos saturados e, como guia de orientação, sugere-se a restrição correspondente à fase $1 \mathrm{da}$ dieta preconizada pelo National Cholesterol Education Program (tab. II) ${ }^{9,11,14,15}$. Do ponto de vista prático, restringe-se o consumo de gordura animal (carne vermelha, leite integral e derivados, manteiga, gema de ovo, embutidos, pele e vísceras de animais, frutos do mar, sorvetes cremosos, óleo de dendê e coco). O consumo de fibras solúveis (frutas, legumes, aveia, cevada) deve ser estimulado. A alimentação básica passa a ser constituída por carnes brancas (aves sem pele e peixes), carne vermelha sem gordura aparente, todos os vegetais, frutas, óleos vegetais (exceto coco e dendê), margarina cremosa, leite desnatado e derivados ${ }^{15}$. Caso a avaliação da resposta à orientação anterior se mostrar insatisfatória após seis meses, maior restrição de colesterol e gorduras é adotada (fase 2, tab. II).

Crianças e adolescentes apresentam habitualmente pequenas modificações de CT e LDL-C com dieta, fato devido não só a aderência inadequada individual e familiar, mas

\begin{tabular}{|l|c|}
\hline \multicolumn{2}{|c|}{ Tabela II - Princípios dietéticos para tratamento } \\
das hipercolesterolemias
\end{tabular}


também a fatores envolvidos na maturação sexual ${ }^{16,17}$. No estudo DISC (Dietary Intervention Study in Children) ${ }^{16}$, realizado em seis centros nos Estados Unidos, 683 pacientes de ambos os sexos, de oito a 10 anos, com valores de LDL-C entre os percentis 75 e 98 , foram orientados a implementar dieta fase 2 . Após três anos, verificou-se que a diminuição de LDL-C foi mais acentuada na fase puberal e de maturidade sexual, tanto no sexo masculino como no feminino, e também foi relacionada ao índice de massa corpóreo. Não ocorreram efeitos deletérios no crescimento e no desenvolvimento sexual, no estado psicológico e nos estoques de ferro.

Crianças e adolescentes que apresentam a forma familiar de hipercolesterolemia apresentam pequena ou nenhuma resposta à orientação dietética ${ }^{11,14}$.

Para a hipertrigliceridemia isolada (tipo IV da classificação de Fredrickson), restringem-se os carboidratos simples (açúcar, mel, balas, sorvetes, bolos, geléia, etc) e, se necessária redução do peso corpóreo, também de carboidratos complexos (farinha, pão, arroz, macarrão, batata, biscoitos). O uso crescente de bebidas alcoólicas deve ser desestimulado. Com essas medidas, níveis ideais de trigliceridemia são facilmente alcançados; entretanto, havendo transgressão, rapidamente se elevam ${ }^{11,14,18}$.

Para a quilomicronemia (tipo I), a redução permanente da ingestão de ácidos graxos saturados e insaturados é recomendada: inicialmente a $20 \mathrm{~g} / \mathrm{dia}$ e, caso não seja alcançado o objetivo, tenta-se redução maior (de até $10 \mathrm{~g} / \mathrm{dia}$ ). $\mathrm{Na}$ prática, restringem-se carnes gordas, leites e seus derivados, óleos de modo geral, e permite-se a adição de óleos com triglicérides de cadeia média no alimento preparado. A resposta é satisfatória ${ }^{11,18}$.

Se a quilomicronemia estiver associada a hipertrigliceridemia (tipo V), a orientação alimentar torna-se mais complexa, pois deve ser restrita a ingestão não só de ácidos graxos saturados e insaturados, como também de carboidratos ${ }^{11,18}$.

Na presença de hiperlipidemia mista (tipos IIb, III), a dieta deve ser pobre em carboidratos, colesterol e ácidos graxos saturados, com aumento da ingestão de insaturados. Para tanto, estimula-se o consumo de frutas, vegetais, carboidratos complexos, fibras e aconselha-se a diminuição do consumo de gordura animal ${ }^{11,15,18}$.

Terapia farmacológica - Associada às medidas anteriormente expostas, a terapia farmacológica dos estados hipercolesterolêmicos deverá ser recomendada após os 10 anos de idade, quando os valores de LDL-C forem: 1) $>190 \mathrm{mg} / \mathrm{dL} ; 2$ ) $\geq 160 \mathrm{mg} / \mathrm{dL}$ se houver antecedentes familiares de DAC prematura ou de presença de dois ou mais fatores de risco; 3 ) $\geq 130 \mathrm{mg} / \mathrm{dL}$ se houver manifestação de doença aterosclerótica ${ }^{9,11,14}$.

Podem ser utilizadas - 1) Resinas sequiestrantes de ácidos biliares: bloqueiam o ciclo êntero-hepático do colesterol e aumentam o número de receptores de LDL. Além de efeitos digestivos indesejáveis, reduzem a absorção de vitaminas lipossolúveis. No Brasil, dispõe-se somente da colestiramina, apresentada em forma de pó, em envelopes de $4 \mathrm{~g}$; a dose média diária é de 8 a 12g, divididas em 2-3 tomadas ${ }^{9,11,14,19}$. Suplementação vitamínica se faz necessária.

2) Vastatinas - São indicadas, em centros de referência, para os portadores de hipercolesterolemia grave poligênica ou familiar heterozigótica. Pequena resposta é observada na forma homozigótica. Elas inibem parcialmente a HMG-CoA redutase, diminuindo a síntese do colesterol, o que leva ao aumento da formação de receptores de LDL. Diminuem também a formação de VLDLe apoB ${ }^{11,14,20,21}$. O emprego de lovastatina e de sinvastatina em crianças e adolescentes com CT $>300 \mathrm{mg} / \mathrm{dL}$ mostrouse eficaz e seguro nas investigações de Ducobu e $\operatorname{col}^{20} \mathrm{e}$ de Sinzinger e $\mathrm{col}^{21}$.

Ducobu e col $^{20}$, em 22 pacientes do sexo masculinoe 10 do feminino e idade inferior a 17 anos, apresentando CT $>300 \mathrm{mg} / \mathrm{dL}$ após dieta, administraram sinvastatina na dose de 5 a $20 \mathrm{~g} /$ dia aos menores de 10 anos e de 10 a $20 \mathrm{~g} / \mathrm{dia}$ aos maiores de 10 anos, durante 24-36 meses. Observaram reduções significativas de CT $(25,5 \%)$, LDL-C (37,3\%), e da relação CT/HDL-C (43,3\%), acompanhadas de elevação de HDL-C (22,5\%). Até a $104^{\mathrm{a}}$ semana de seguimento, não foram observadas alterações enzimáticas significativas; o desenvolvimento e crescimento foram mantidos.

Sinzinger e $\mathrm{col}^{21}$ administraram durante 208 semanas também em hipercolesterolêmicos com CT >300mg/dLe idade inferior a 13 anos, lovastatina na dose de $20 \mathrm{mg} / \mathrm{dia}$ isoladamente ou associada a 8g/dia de colestiramina. Reduções significativas de CT e LDL-C ocorreram, porém foram mais acentuadas na presença da associação. Essas reduções não foram acompanhadas por alterações enzimáticas significativas e nem do desenvolvimento e crescimento.

Na presença de quadro clínico-laboratorial compatível com hipercolesterolemia familiar homozigótica, tem sido cogitado o uso de sinvastatina em doses elevadas (80 a $160 \mathrm{mg} / \mathrm{dia}$ ) ou de atorvastatina ${ }^{22,23}$.

No Brasil, já estão sendo comercializadas a lovastatina, a sinvastatina, a pravastatina, a fluvastatina, a atorvastatina e a cerivastatina. Entretanto, a experiência com estas drogas no tratamento dos estados hipercolesterolêmicos de crianças e adolescentes ainda é pequena em nosso meio.

3) Ácido nicotínico e derivados têm sido empregado em centros de referência dos Estados Unidos e Europa em número limitado de pacientes. Diminuem a formação de VLDL, apo B e, LDL ${ }^{11,14}$.

Não se empregam fármacos hipolipemiantes nos estados hipertrigliceridêmicos ${ }^{11,14}$. Entretanto, nessas situações, podem ser administrados ácidos graxos ômega 3 como suplemento alimentar ${ }^{11}$.

Medidas alternativas - Somente são adotadas em centros de referência para os hipercolesterolêmicos heterozigóticos ou homozigóticos: além de complexas, seu custo é elevado e exigem maiores cuidados médicos. São elas: afereses (plasmaferese, LDL-aferese), transplante de fígado, terapia gênica. A plasmaferese também pode ser indicada para os raros casos de quilomicronemia acentuada quando há risco de pancreatite ${ }^{9,11,14,24,25}$. 


\section{Considerações finais}

À semelhança de outros países, a maior parte das dislipidemias em crianças e adolescentes brasileiros está relacionada a hábitos alimentares e costumes inadequados individuais e familiares. Orientações relacionadas à adoção de hábitos alimentares saudáveis, realização de exercícios físi- cos, controle do peso corpóreo, abandono do tabagismo devem ser dadas individualmente e às famílias. Palestras neste sentido também devem ser feitas nas escolas e clubes. Entretanto, particular atenção deve ser dada às crianças e adolescentes, parentes diretos de coronarianos. Terapia farmacológica e medidas alternativas são reservadas para os casos potencialmente mais graves.

\section{Referências}

1. McGill HC Jr, Geer JC, Strong JP - Natural history of human atherosclerotic lesions. In: Sandler M, Bourne GH (ed) - Atherosclerosis and Its Origin. New York: Academic Press, 1963: 39-65.

2. Berenson GS, Wattigney WA, Tracy RE et al - Atherosclerosis of the aorta and coronary arteries and cardiovascular risk factors in persons aged 6 to 30 years and studied at necropsy (The Bogalusa Heart Study). Am J Cardiol 1992; 70 : 851-8.

3. McGill HC Jr, McMahan CA, Malcom GT et al - Effects of serum lipoproteins and smoking on atherosclerosis in young men and women. The PDAY Research Group. Pathobiological determinants of atherosclerosis in youth. Arterioscl Thromb Vasc Biol 1997; 17: 95-106.

4. Sprecher DL, Schaeffer EJ, Kent KM et al - Cardiovascular features of homozygous familial hypercholesterolemia: analysis of 16 patients. Am J Cardiol 1984; 54: 20-30.

5. Celermajer DS, Sorensen KE, Gooch VM et al - Noninvasive detection of endothelial dysfunction in children and adults at risk of atherosclerosis. Lancet 1992; 340: 1111-15.

6. Webber LS, Srinivasan SR, Wattigney WA et al - Tracking of serum lipids and lipoproteins from childhood to adulthood. Am J Epidemiol 1991; 133: 884-99.

7. Lauer RM, Clarke WR - Use of cholesterol measurement in childhood for the prediction of adult hypercholesterolemia. The Muscatine Study. JAMA 1990; 264: 3034-8.

8. Cortner JA, Coates PM, Gallagher PR - Prevalence and expression of familial combined hyperlipidemia in childhood. J Pediatr 1990; 116: 514-19.

9. Highlights of the Report of the Expert Panel on Blood Cholesterol Levels in Children and Adolescents. Am Fam Phys 1992; 45: 2127-36.

10. Kwiterovich Jr PO - Beyond Cholesterol. The Johns Hopkins Complete Guide for avoiding heart disease. Baltimore: The Johns Hopkins University Press, 1989: 1-395.

11. Consenso Brasileiro sobre Dislipidemias. Detecção, Avaliação e Tratamento. Arq Bras Cardiol 1996; 67: 1-16.

12. Gerber RS - Fatores de risco na infância: estudo epidemiológico. Dissertação de mestrado. Curso de Pós-Graduação em Medicina - Pediatria. Universidade do Rio Grande do Sul, Porto Alegre, 1992.
13. Forti N, Giannini SD, Diament $\mathrm{J}$ et al - Fatores de risco para doença arterial coronariana em crianças e adolescentes filhos de coronariopatas jovens. Arq Bras Cardiol 1996; 66: 119-23.

14. Kwiterovich PO - Prevention of coronary disease starting in childhood: what risk factors should be identified and treated? Coronary artery disease 1993; 4 : 611-30.

15. Costa RP, Martinez TLR - Terapia nutricional na hipercolesterolemia. Rev Soc Cardiol Estado de São Paulo 1997; 4: 485-9.

16. Kwiterovich PO, Barton BA, McMahon RP et al - Effects of diet and sexual maturation on low-density lipoprotein cholesterol during puberty. The Dietary Intervention Study in Children (DISC). Circulation 1997; 96 : 2526-33.

17. McGill HC - Nutrition in early life and cardiovascular disease. Curr Opin Lipidol 1998; 9: 23-7.

18. Diament J, Marcilio CS - Tratamento dietético das hipertrigliceridemias. Rev Soc Cardiol Estado de São Paulo 1997; 4: 485-9.

19. Farah R, Kwiterovich PO, Neil CA - A study of the dose effect of cholestiramine in children and young adult with familial hypercholesterolemia. Lancet 1977; 1: 59-63.

20. Ducobu J, Brasseur D, Chaudron JM et al - Simvastatin use in children. Lancet 1992; 339: 1488 .

21. Sinzinger H, Schimid P, Pirich C et al - Treatment of hypercholesterolemia in children. Lancet 1992; 340: 548-9.

22. Raal F, Pilcher G, Illingworth DR et al - Expanded-dose simvastatin is effective in homozygous familial hypercholesterolaemia. Atherosclerosis 1997; 249: 56.

23. Marais AD, Naoumova RP, Firth JC et al - Decreased production of low density lipoprotein by atorvastatin after apheresis in homozygous familial hypercholesterolemia. J Lipid Res 1997; 38: 2071-78.

24. Hoeg JM - Pharmacological and surgical treatment of dyslipidemic children and adolescents. Ann NY Acad Sci 1991; 623: 275-84.

25. Uavy R, Zwiener RS, Philips MJ et al - Treatment of children with homozygous familial hypercholesterolemia safety and efficacy of low density lipoprotein apheresis. J Ped 1992; 120: 892-8. 